\title{
Sequential Therapy for First-Line Helicobacter pylori Eradication: 10- or 14-Day Regimen?
}

\author{
Angelo Zullo ${ }^{1}$, Giulia Fiorini ${ }^{2}$, Giuseppe Scaccianoce ${ }^{3}$, Piero Portincasa ${ }^{3}$, Vincenzo De Francesco ${ }^{4}$, Roberto Vassallo ${ }^{5}$, \\ Flavia Urban ${ }^{6}$, Fabio Monica ${ }^{6}$, Giuseppe Mogavero ${ }^{7}$, Arnaldo Amato ${ }^{7}$, Dino Vaira ${ }^{2}$
}

1) Gastroenterology and

Digestive Endoscopy, 'Nuovo

Regina Margherita' Hospital,

Rome,

2) Department of Medical and Surgical Sciences, 'S. Orsola'

Hospital, University of

Bologna, Bologna

3) Internal Medicine A. Murri, Department of Biomedical

Sciences \& Human Oncology,

University of Bari, Bari

4) Section of Gastroenterology,

Department of Medical

Sciences, University of Foggia,

Foggia

5) Gastroenterology and

Digestive Endoscopy;

"Buccheri la Ferla,

Fatebenefratelli” Hospital,

Palermo

6) Gastroenterology and

Digestive Endoscopy,

Academic Hospital Cattinara,

Trieste

7) Gastroenterology Unit,

'Valduce' Hospital, Como, Italy

\footnotetext{
Address for correspondence: Angelo Zullo

Gastroenterologia ed Endoscopia Digestiva,

Ospedale Nuovo Regina

Margherita, Via Emilio

Morosini, 3000153 Roma,

Italy

angelozullo66@yahoo.it
}

Received: 29.10.2018

Accepted: 14.01 .2019

\section{ABSTRACT}

Background \& Aim: Standard 10-day sequential therapy is advised as first-line therapy for Helicobacter pylori (H. pylori) eradication by current Italian guidelines. Some data suggested that a 14-day regimen may achieve higher eradication rates. This study compared the efficacy of sequential therapy administered for either 10- or 14-days.

Methods: This prospective, multicenter, open-label study enrolled patients with $H$. pylori infection without previous treatment. Patients were receiving a sequential therapy for either 10 or 14 days with esomeprazole $40 \mathrm{mg}$ and amoxicillin $1 \mathrm{~g}$ (5 or 7 days) followed by esomeprazole $40 \mathrm{mg}$, clarithromycin $500 \mathrm{mg}$ and tinidazole $500 \mathrm{mg}$ ( 5 or 7 days), all given twice daily. Bacterial eradication was checked using ${ }^{13} \mathrm{C}$-urea breath test. Eradication cure rates were calculated at both Intention-to-treat (ITT) and per-protocol (PP) analyses. Results: A total of 291 patients were enrolled, including 146 patients in 10-day and 145 in the 14-day regimen. The eradication rates were $87 \%(95 \% \mathrm{CI}=81.5-92.4)$ and $90.3 \%(95 \% \mathrm{CI}=85.5-95.1)$ at ITT analysis with the 10 - and 14-day regimen, respectively, and $92.7 \%(95 \% \mathrm{CI}=88.3-97)$ and $97 \%(95 \% \mathrm{CI}=94.2-99.9)$ at $\mathrm{PP}$ analysis ( $\mathrm{p}=0.37$ ). Among patients, who earlier had interrupted therapy, bacterial eradication was achieved in 8 out of 9 who completed the first therapy phase and performed at least $\geq 3$ days of triple therapy in the second phase.

Conclusion: This study found that both 10- and 14-day sequential therapies achieved a high eradication rate for first-line H. pylori therapy in clinical practice.

Key words: Helicobacter pylori - sequential therapy - eradication.

Abbrevations: CI: confidence intervals; ITT: intention to treat; PP: per protocol; SD: standard deviation; UBT: urea breath test.

\section{INTRODUCTION}

Despite the fact that its prevalence is decreasing, $H$. pylori is still a widespread infection causing benign and malignant diseases [1]. Unfortunately, no therapy regimen achieves bacterial eradication in all patients, the treatment for the infection remaining challenging [2]. Current Italian guidelines suggested the use of either 14day triple therapy or 10-day sequential therapy for first-line therapy [3]. However, data of different Italian studies showed that prolonged triple therapy achieved a success rate of only
$77 \%$ in 619 patients, even when a high-dose proton pump inhibitor was used [4-6]. On the contrary, a high success rate was obtained following 10-day sequential therapy in Italy [7], as well as in several other countries [8]. However, a recent network meta-analysis showed that sequential therapy for 14 days achieves the highest effectiveness as compared to all the other regimens [9]. Although helpful, data of a network metaanalysis cannot overcome the need to test a specific therapy in a particular region before selecting the best available treatment [10]. Therefore, we designed a prospective study to compare the efficacy of sequential therapy administered for either 10- or 14-days regimen in clinical practice.

\section{PATIENTS AND METHODS}

\section{Patients}

This was a prospective, multicenter, open-label study enrolling adult patients referred for dyspeptic symptoms in the Endoscopy Units of the seven involved centres. All patients 
with $H$. pylori diagnosed at the histological examination of antral and gastric body biopsies, never previously treated for the infection, were invited to participate. Patients with known allergy to penicillin or macrolides were excluded, as well as those with relevant diseases. Bacterial culture with antibiotic susceptibility testing on agar was performed in a single centre (Bologna), by using commercial selective medium Pylori Agar as routinely performed [7]. Patients received a sequential therapy for either 10 or 14 days with esomeprazole $40 \mathrm{mg}$ and amoxicillin $1 \mathrm{~g}$ (5 or 7 days) followed by esomeprazole $40 \mathrm{mg}$, clarithromycin $500 \mathrm{mg}$ and tinidazole $500 \mathrm{mg}$ (5 or 7 days). All drugs were given twice daily. A pre-fixed, computer generated list was used for randomization in each centre. At the end of therapy, the self-reported compliance and side-effects were assessed by a personal questionnaire. Following 6-8 weeks, standard ${ }^{13} \mathrm{C}$-urea breath test (UBT) was performed to evaluate bacterial eradication rates. The study was performed according to the guidelines for Good Clinical Practice and the Declaration of Helsinki (1996 version, amended October 2000). Since no experimental drugs were administered, no adjunctive costs or procedures for the patients were required, no identification of patients was allowed, and no funds were received, our Investigational Review Board waived formal review and approval, deeming the study to be an extension of existing clinical practice.

\section{Statistical analysis}

The sample size was calculated as for a 'non-inferiority' study. By expecting a $90 \%$ eradication rate following the 10 -day regimen and a $95 \%$ with the 14 -day therapy, with a power of $90 \%$ and significance level of 5\%, a total of 236 patients $(118$ per arm) should have been enrolled. When considering a $10 \%$ drop-out rate, data of 260 patients ( 130 per arm) needed to be included. The eradication rates with $95 \%$ confidence intervals (CI) were calculated at both 'intention-to-treat' (ITT) (all patients who accepted to participate) and at 'per-protocol' (PP) (all patients who effectively performed therapy and UBT control) analyses. Patients who completed at least the first phase of therapy (i.e. 5 or 7 day with dual therapy) underwent UBT control, whilst those who performed a shorter therapy were considered as drop-outs. Before pooling data, a statistical comparison was performed among data from different centres to rule out heterogeneity. The Chi-squared test and the Fisher's exact test were used as appropriate, and a p level less than 0.05 was considered statistically significant.

\section{RESULTS}

The study enrolled 291 consecutive patients (M/F: 122/169; mean age: $49.3 \pm 12.6$ years $)$. At endoscopy, 8 patients had duodenal ulcer, 3 gastric ulcer, 24 erosions (gastric or duodenal), whilst no endoscopic lesions were detected in the remaining 252 patients (Table I). Overall, 146 patients received the 10-day therapy and 145 the 14-day regimen, but 9 and 10 patients, respectively, were lost at follow-up. Following the 10day regimen the infection was cured in 127 patients accounting for $87 \%(95 \% \mathrm{CI}=81.5-92.4)$ eradication rate at ITT and $92.7 \%$ $(95 \% \mathrm{CI}=88.3-97)$ eradication rate at PP analyses. Following the 14-day regimen, the infection was cured in 131 patients accounting for $90.3 \%(95 \% \mathrm{CI}=85.5-95.1)$ eradication rate at ITT and $97 \%(95 \% \mathrm{CI}=94.2-99.9)$ eradication rate at PP analyses. Although the 14-day regimen achieved a $+3.3 \%$ (at

Table I. Demographic and clinical characteristics of enrolled patients

\begin{tabular}{lccc}
\hline & $\begin{array}{c}\text { 10-day } \\
\text { therapy }\end{array}$ & $\begin{array}{c}14 \text {-day } \\
\text { therapy }\end{array}$ & $\begin{array}{c}\mathrm{P} \\
\text { value }\end{array}$ \\
\hline Number of patients & 146 & 145 & - \\
Age (mean \pm SD) (years) & $48.2 \pm 13.2$ & $49.9 \pm 11.6$ & 0.48 \\
Sex (male/female) & $63 / 83$ & $59 / 86$ & 0.29 \\
Upper endoscopy & & & 0.10 \\
$\quad$ - Duodenal ulcer & 4 & 5 & \\
- Gastric ulcer & 2 & 1 & \\
$\quad$ - Gastroduodenal erosions & 11 & 13 & \\
$\quad$ - No lesions & 129 & 136 & \\
\hline
\end{tabular}

Table II. Cure rate at intention-to-treat (ITT) and per-protocol (PP) analyses in different

\begin{tabular}{lcccccc}
\hline Center & $\begin{array}{l}\text { Therapy } \\
\text { regimen }\end{array}$ & Enrolled & Drop-out & Not cured & ITT (\%) & PP (\%) \\
\hline Bari & 10 & 26 & 3 & 1 & $22 / 26(84.6)$ & $22 / 23(95.6)$ \\
& 14 & 28 & 1 & 0 & $27 / 28(96.4)$ & $27 / 27(100)$ \\
\hline Bologna & 10 & 40 & 2 & 2 & $36 / 40(90)$ & $36 / 38(94.7)$ \\
& 14 & 40 & 2 & 2 & $36 / 40(90)$ & $36 / 38(94.7)$ \\
\hline Como & 10 & 7 & 1 & 0 & $6 / 7(85.7)$ & $6 / 6(100)$ \\
& 14 & 6 & 1 & 0 & $5 / 6(83.3)$ & $5 / 5(100)$ \\
\hline Foggia & 10 & 20 & 1 & 3 & $16 / 20(80)$ & $16 / 19(84.2)$ \\
& 14 & 20 & 1 & 0 & $19 / 20(95)$ & $19 / 19(100)$ \\
\hline Palermo & 10 & 11 & 0 & 1 & $10 / 11(90.9)$ & $10 / 11(90.9)$ \\
& 14 & 10 & 0 & 1 & $9 / 10(90)$ & $9 / 10(90)$ \\
\hline \multirow{2}{*}{ Roma } & 10 & 32 & 1 & 2 & $29 / 32(90.6)$ & $29 / 31(93.5)$ \\
& 14 & 31 & 2 & 1 & $28 / 31(90.3)$ & $28 / 29(96.6)$ \\
\hline Trieste & 10 & 10 & 1 & 1 & $8 / 10(80)$ & $8 / 9(88.9)$ \\
& 14 & 10 & 3 & 0 & $7 / 10(70)$ & $7 / 7(100)$ \\
\hline Total & 10 & 142 & 9 & 10 & $127 / 146(87)$ & $127 / 137(92.7)$ \\
& 14 & 144 & 10 & 4 & $131 / 145(90.3)$ & $131 / 135(97)$ \\
\hline
\end{tabular}


ITT analysis) and $+4.3 \%$ (at PP) cure rate as compared to the 10 -day regimen, no statistically significant difference emerged among groups (Table II). Data of eradication rates according to the pattern of antibiotic resistance are provided in Table III.

A total of $31(21.2 \%)$ patients in the 10 -day therapy complained of side-effects, causing early therapy interruption in 3 cases. In the 14-day regimen, 39 (26.9\%) patients reported side-effects, and 8 failed to complete therapy. Data of patients who earlier interrupted therapy were provided in Table IV. As shown, bacterial eradication was achieved in 8 out of 9 patients who completed the first therapy phase and performed at least $\geq 3$ days of triple therapy in the second phase. Overall, the most frequently complained side-effects without the need to withdraw therapy were: nausea (22 cases), bad taste (16 cases), abdominal pain (15 cases) and mild diarrhoea (6 cases).

Table III. Eradication according to the antibiotic resistance pattern peformed in single centre (Bologna)

\begin{tabular}{lccc}
\hline Resistance pattern & $\begin{array}{c}\text { 10-day therapy } \\
(\mathrm{N}=22)\end{array}$ & $\begin{array}{c}\text { 14-day therapy } \\
(\mathrm{N}=32)\end{array}$ & Total \\
\hline Cla-S Met-S & $12 / 12$ & $\begin{array}{c}13 / 13 \\
(1 \text { drop out })\end{array}$ & $25 / 25(100 \%)$ \\
Cla-R Met-S & 2 drop-out & $4 / 4$ & $4 / 4(100 \%)$ \\
Cla-S Met-R & $3 / 3$ & $8 / 8$ & $11 / 11(100 \%)$ \\
Cla-R Met-R & $5 / 5$ & $3 / 5$ & $8 / 10(80 \%)$ \\
\hline
\end{tabular}

Cla: clarithromycin; Met: metronidazole; S: susceptible; R: resistant.

Table IV. Outcome in patients who earlier interrupted therapy due to side-effects

\begin{tabular}{cccc}
\hline Therapy assigned & Days of herapy & Outcome & Side-effects \\
\hline 14-day & $7+6$ & Cured & Nausea \\
14-day & $7+6$ & Cured & Abdominal pain \\
14-day & $7+5$ & Cured & Nausea \\
14-day & $7+5$ & Cured & Abdominal pain \\
14-day & $7+4$ & Cured & Vomiting \\
14-day & $7+3$ & Cured & Abdominal pain \\
14-day & $7+1$ & Cured & Vomiting \\
14-day & $3+0$ & Drop-out & Urticaria \\
10-day & $5+3$ & Cured & Diarrhoea \\
10-day & $5+3$ & Not cured & Nausea \\
10-day & $2+0$ & Drop-out & Vomiting \\
\hline
\end{tabular}

\section{DISCUSSION}

Curing H. pylori infection still remains a challenge in clinical practice. The 10-day sequential therapy, suggested as first-line therapy in the current Italian guidelines [3], has been proven to be effective in several countries, achieving eradication rates as high as $91.1 \%-93.7 \%$ in Italy $[7,11], 92.5-95 \%$ in Turkey [12,13], 94.2\% in Slovenia [14], 90\% in Portugal [15], 90\% in Belgium [16], 95.9\% in Israel [17], 94\% in Thailand [18], $91.9 \%$ in Taiwan [19], $90.3 \%$ in Singapore [20], and $88.6 \%$ in the United Arab Emirates [21]. In detail, the 10-day sequential therapy was found to be equally effective - but cheaper - than quadruple therapy with Pylera (each pill containing $140 \mathrm{mg}$ bismuth subcitrate potassium, $125 \mathrm{mg}$ metronidazole, and $125 \mathrm{mg}$ tetracycline) for first-line therapy $[22,23]$. However, a recent network meta-analysis showed that the 14-day sequential therapy achieved a higher eradication rate as compared to all the other first-line regimens [8]. Therefore, we performed a 'head to head' comparison between sequential regimens administered for either 10 or 14 days in clinical practice. Overall, data found that both therapies achieved high $H$. pylori eradication rates (87\%-90.3\% at ITT and $92.7 \%-97 \%$ at PP), without a statistically significant difference between treatments. In detail, the longer regimen tended to increase $(+3 \%,+4 \%)$ the cure rate as compared to the standard 10-day therapy, but the therapeutic gain was not statistically significant, and the cost was increased (42.84 vs. 30.6 Euros in Italy). Therefore, it would appear that prolonging the sequential therapy is not cost-effective, at least in Italy. However, testing the efficacy of the 14-day sequential therapy could be useful in those areas where the standard 10 -day regimen was proven to be not highly effective [4]. The high efficacy of both sequential regimens is noteworthy when considering the elevated levels of primary resistance towards both clarithromycin and metronidazole in $H$. pylori isolates found in Italy [24]. We found that high eradication rates were achieved when bacterial strains harboured a single resistance towards either clarithromycin or metronidazole, with an $80 \%$ cure rate even in those strains with dual resistance. A recent study found that bacterial resistance becomes relevant in vivo only when clarithromycin-resistant and metronidazoleresistant strains have high MIC values for at least one of these antibiotics [25].

In the past, several attempts were performed to further improve the efficacy of standard 10-day sequential therapy, by increasing duration or substituting clarithromycin with either levofloxacin or tetracycline [26]. However, no modified therapy schedule was found to be consistently superior to the standard 10-day regimen. In detail, a levofloxacin-based sequential therapy achieved high eradication rate in an Italian study where primary levofloxacin resistance was very low [27], but distinctly lower cure rates were achieved in several studies in other countries [25].

Noteworthy, by considering patients who early interrupted therapy in the present study, we observed that the infection was cured in all cases that completed the first 7 days dual therapy and at least 3 days of successive triple therapy. Although preliminary, these data suggest that the efficacy of a modified 'seven plus three'-day sequential therapy could be tested in a pilot study.

\section{CONCLUSION}

Our study found that both the 10- and 14-day sequential therapies achieved a high eradication rate as first-line $H$. pylori therapy in clinical practice, with no statistical difference between the two treatments.

Conflicts of interest: No conflicts of interest.

Authors' contributions: A.Z. and D.V. conceived the study. A.Z. and G.F. analysed data and prepared the manuscript. A.Z., G.F., G.S., 
P.P. V.de F. R.V., F.U, F.M, G.M., A.A and D.V. enrolled patients and critically reviewed the manuscript.

\section{REFERENCES}

1. Malfertheiner P, Megraud F, O'Morain CA, et al. Management of Helicobacter pylori infection-the Maastricht V/Florence Consensus Report. Gut 2017;66:6-30. doi: 10.1136/gutjnl-2016-312288

2. Fiorini G, Zullo A, Gatta L, et al. Newer agents for Helicobacter pylori eradication. Clin Exp Gastroenterol 2012;5:109-112. doi: 10.2147/CEG. S25422

3. Zagari RM, Romano M, Ojetti V, et al. Guidelines for the management of Helicobacter pylori infection in Italy: The III Working Group Consensus Report 2015. Dig Liver Dis 2015;47:903-912. doi: 10.1016/j. dld.201506.010

4. De Francesco V, Bellesia A, Ridola L, Manta R, Zullo A. First-line therapies for Helicobacter pylori eradication: a critical reappraisal of updated guidelines. Ann Gastroenterol 2017;30:373-379. doi: 10.20524/ aog.2017.0166

5. Zullo A, Severi C, Vannella L, Hassan C, Sbrozzi-Vanni A, Annibale B. Role of gastritis pattern on Helicobacter pylori eradication. Intern Emerg Med 2012;7:517-522. doi:10.1007/s11739-011-0730-4

6. De Francesco V, Ridola L, Hassan C, et al. Two-week triple therapy with either standard or high-dose esomeprazole for first-line $\mathrm{H}$. pylori eradication. J Gastrointestin Liver Dis 2016;25:147-150. doi:10.15403/ jgld.2014.1121.252.2w3

7. Gatta L, Scarpignato C, Fiorini G, et al. Impact of primary antibiotic resistance on the effectiveness of sequential therapy for Helicobacter pylori infection: lessons from a 5-year study on a large number of strains. Aliment Pharmacol Ther 2018;47:1261-1269. doi:10.1111/apt.14597

8. Gatta L, Vakil N, Vaira D, Scarpignato C. Global eradication rates for Helicobacter pylori infection: systematic review and meta-analysis of sequential therapy. BMJ 2013;347:f4587. doi:10.1136/bmj.f4587

9. Yeo YH, Shiu SI, Ho HJ, et al. First-line Helicobacter pylori eradication therapies in countries with high and low clarithromycin resistance: a systematic review and network meta-analysis. Gut 2017;67:20-27. doi:10.1136/gutjnl-2016-311868

10. Braden B. The best and worst treatments for Helicobacter pylori. BMJ 2015;351:h4146. doi:10.1136/bmj.h4146

11. De Francesco V, Pontone S, Bellesia A, et al. Quadruple, sequential, and concomitant first-line therapies for $\mathrm{H}$. pylori eradication: a prospective, randomized study. Dig Liver Dis 2018;50:139-441. doi:10.1016/j. dld.2017.10.009

12. Kefeli A, Basyigit S, Yeniova AO, Kefeli TT, Aslan M, Tanas O. Comparison of three different regimens against Helicobacter pylori as a first-line treatment: a randomized clinical trial. Bosn J Basic Med Sci 2016;16:52-57. doi:10.17305/bjbms.2016.660

13. Harmandar F, İlikhan SU, Üstüündağn Y, Harmandar O. The efficacy of sequential therapy in eradication of Helicobacter pylori in Turkey. Niger J Clin Pract 2017;20:616-621. doi:10.4103/11193077.196991

14. Tepeš B, Vujasinović $M$, Šeruga $M$, Stefanovič $M$, Forte A, Jeverica S. Randomized clinical trial comparing 10-day sequential, 7-day concomitant and 7-day standard triple therapies for Helicobacter pylori eradication. Eur J Gastroenterol Hepatol 2016;28:676-683. doi:10.1097/ MEG.0000000000000590

15. Branquinho D, Almeida N, Gregório C, et al. Levofloxacin or clarithromycin-based quadruple regimens: what is the best alternative as first-line treatment for Helicobacter pylori eradication in a country with high resistance rates for both antibiotics? BMC Gastroenterol 2017;17:31. doi:10.1186/s12876-017-0589-6

16. Kotilea K, Mekhael J, Salame A, et al. Eradication rate of Helicobacter pylori infection is directly influenced by adherence to therapy in children. Helicobacter 2017;22: e12383. doi:10.1111/hel.12383

17. Schmilovitz-Weiss H, Shalev T, Chechoulin Y, et al. High eradication rates of Helicobacter pylori infection following sequential therapy: the Israeli experience treating naïve patients. Helicobacter 2011;16:229-233. doi:10.1111/j.1523-5378.2011.00834.x

18. Phiphatpatthamaamphan K, Vilaichone RK, Siramolpiwat S, et al. Effect of IL-1 polymorphisms, CYP2C19 genotype and antibiotic resistance on Helicobacter pylori eradication comparing between 10-day sequential therapy and 14-day standard triple therapy with four-times-daily-dosing of amoxicillin in Thailand: a prospective randomized study. Asian Pac J Cancer Prev 2016;17:1903-1907.

19. Liou JM, Chen CC, Chang CY, et al. Sequential therapy for 10 days versus triple therapy for 14 days in the eradication of Helicobacter pylori in the community and hospital populations: a randomized trial. Gut 2016;65:1784-1792. doi:10.1136/gutjnl-2015-310142

20. Ang TL, Fock KM, Song M, et al. Ten-day triple therapy versus sequential therapy versus concomitant therapy as first-line treatment for Helicobacter pylori infection. J Gastroenterol Hepatol 2015;30:11341139. doi:10.1111/jgh.12892

21. Abuhammour A, Dajani A, Nounou M, Zakaria M. Standard triple therapy versus sequential therapy for eradication of Helicobacter pylori in treatment naïve and retreat patients. Arab J Gastroenterol 2016;17:131-136. doi:10.1016/j.ajg.2016.07.001

22. Fiorini G, Zullo A, Saracino IM, Gatta L, Pavoni M, Vaira D. Pylera and sequential therapy for first-line Helicobacter pylori eradication: a culture-based study in real clinical practice. Eur J Gastroenterol Hepatol 2018;30:621-625.

23. Di Ciaula A, Scaccianoce G, Venerito M, et al. Eradication rates in Italian subjects heterogeneously managed for Helicobacter pylori infection. Time to abandon empiric treatments in Southern Europe. J Gastrointestin Liver Dis 2017;26:129-137. doi:10.15403/jgld.2014.1121.262.itl

24. Fiorini G, Zullo A, Saracino IM, Pavoni M, Vaira D. Antibiotic resistance pattern of Helicobacter pylori strains isolated in Italy during 2010-2016. Scand J Gastroenterol 2018;53:661-664. doi:10.1080/00365521.2018.14 64596

25. De Francesco V, Zullo A, Fiorini G, Saracino IM, Pavoni M, Vaira D. Role of MIC levels of resistance to clarithromycin and metronidazole in Helicobacter pylori eradication. J Antimicrob Chemother $2018 \mathrm{Nov}$ 26. doi: $10.1093 / \mathrm{jac} / \mathrm{dky}-469$

26. Zullo A, De Francesco V, Hassan C, et al. Modified sequential therapy regimens for Helicobacter pylori eradication: a systematic review. Dig Liver Dis 2013;45:18-22. doi: 10.1016/j.dld.2012.08.025

27. Romano M, Cuomo A, Gravina AG, et al. Empirical levofloxacincontaining versus clarithromycin-containing sequential therapy for Helicobacter pylori eradication: a randomised trial. Gut 2010;59:14651470. doi: 10.1136/gut.2010.215350 